\title{
Langmuir
}

pubs.acs.org/Langmuir

(C) 2010 American Chemical Society

\section{Structures and Phase Behavior in Mixtures of Charged Colloidal Spheres and Platelets}

\author{
Dzina Kleshchanok, ${ }^{*}{ }^{\dagger}$ Andrei V. Petukhov, ${ }^{\dagger}$ Peter Holmqvist, ${ }^{\dagger}$ Dmytro V. Byelov, ${ }^{\dagger}$ and \\ Henk N. W. Lekkerkerker ${ }^{\dagger}$ \\ ${ }^{\dagger}$ Van 't Hoff Laboratory, Debye Institute for Nanomaterials Science, Utrecht University, The Netherlands, and \\ *Soft Matter Group, Institute of Solid State Research (IFF), Forschungszentrum Juelich, Germany
}

Received May 12, 2010. Revised Manuscript Received July 1, 2010

\begin{abstract}
The experimental phase diagram for aqueous mixtures of charged gibbsite platelets and silica spheres is presented. The platelets are $95 \mathrm{~nm}$ in diameter, and the diameter ratio between the spheres and the platelets is 0.18 . Here the spheres are acting as depletants in the mixtures perturbing the phase behavior of the pure platelet suspensions. An important finding is that a large isotropic/columnar coexistence region has been identified in the phase diagram, which appeared already at low concentrations of the platelets. Microradian X-ray diffraction measurements revealed the structure of the liquid crystalline phases and the orientational order of platelets. An interesting observation is that in the columnar phase the silica spheres are located between the columnar stacks. All samples were in equilibrium because sedimentation did not affect the system because of the relatively small size of the colloidal particles and the charges present at their surfaces.
\end{abstract}

\section{Introduction}

Mixing colloids of different type and shape occurs frequently in food technology, biological systems, construction, and engineering. ${ }^{1}$ For example, natural clays from the smectite family are widely used as components of drilling fluids for tunnel constructions and the oilmining industry. ${ }^{2,3}$ The rheological and structuring effects of these drilling fluids can be significantly enhanced by mixing clays with distinctly different colloidal particles. Reported examples include caolinite/silica ${ }^{4,5}$ and hectorite/ $/$ silica $^{6}$ mixtures. To be able to control and manipulate the mechanical properties of these suspensions, information about their structure and phase behavior is essential.

Smectite clay particles are thin and somewhat flexible platelets with a large polydispersity in both size and shape. ${ }^{7}$ These features make smectite clays difficult to control and to describe theoretically. In the present work, we therefore chose a simpler experimental model system, which was developed in the van 't Hoff laboratory in Utrecht. ${ }^{8}$ The colloidal particles are inorganic gibbsite $\left(\gamma-\mathrm{Al}(\mathrm{OH})_{3}\right)$ platelets with pronounced hexagonal shape and the thickness, $L$, significantly smaller than the diameter, $D(L \ll D)$. In typical, the $L / D$ ratio can be adjusted during the synthesis procedure between 0.03 and 0.5 . Gibbsite platelets can be sterically ${ }^{9}$ or electrostatically stabilized. ${ }^{10}$

Previously, gibbsite particles were used to study the phase behavior of platelet suspensions. ${ }^{11}$ In general, the phase behavior

\footnotetext{
*Corresponding author. E-mail: D.Kleshchanok@uu.nl.

(1) Russel, W. B.; Saville, D. A.; Schowalter, W. R. Colloidal Dispersions; Cambridge University Press: Cambridge, U.K., 1989; p 525.

(2) Maitland, G. C. Curr. Opin. Colloid Interface Sci. 2000, 5, 301-311.

(3) Murray, H. H. Appl. Clay Sci. 2000, 17, 207-221.

(4) Baird, J. C.; Walz, J. Y. J. Colloid Interface Sci. 2006, 297, 161-169.

(5) Baird, J. C.; Walz, J. Y. J. Colloid Interface Sci. 2007, 306, 411-420.

(6) ten Brinke, A. J. W.; Bailey, L.; Lekkerkerker, H. N. W.; Maitland, G. C.

Soft Matter 2008, 4, 337-348.

(7) van Olpen, H. An Introduction to Clay Colloid Chemistry; Interscience: New York, 1963.

(8) Wierenga, A. M.; Lenstra, T. A. J.; Philipse, A. P. Colloids Surf., A 1998, 134, 359-371.

(9) van der Kooij, F. M.; Lekkerkerker, H. N. W. J. Phys. Chem. B 1998, 102, 7829-7832.

(10) van der Beek, D.; Lekkerkerker, H. N. W. Europhys. Lett. 2003, 61, 702-707.

(11) Davidson, P.; Gabriel, J.-C. P. Curr. Opin. Colloid Interface Sci. 2005, 9, 377-383.
}

of platelet-like particles is richer than that of spheres because of their additional orientational degree of freedom. Gibbsite platelets, both sterically and electrostatically stabilized, are able to form liquid crystalline phases, such as nematic and columnar phases. ${ }^{9,10,12-14}$ The nematic state is characterized by particle orientational order, whereas the columnar phase additionally possesses positional order in two dimensions. ${ }^{15}$

Mixing colloidal particles with another component, differing in size or shape, leads to the well-known effect of depletion attraction. ${ }^{16}$ At sufficiently high concentrations of the colloid and the depletant, phase separation will occur because of the gain in free volume available to the depletant. Mixtures of colloidal platelets and polymer depletants were studied both theoretically ${ }^{17-19}$ and experimentally. ${ }^{20-22}$ Theoretical calculations for mixtures of hard discs and small polymers predict the existence of isotropic, nematic, and columnar phases at low polymer concentrations and a significant broadening of the isotropic/columnar $(\mathrm{I}+\mathrm{C})$ coexistence region at larger depletant concentrations. ${ }^{17,18}$ Experimental phase diagrams show, however, a richer phase behavior. For instance, a four-phase coexistence region was found, involving an isotropic gas and a liquid phase together with the nematic and columnar phases. ${ }^{22}$ Wensink et al. ${ }^{18}$ theoretically explained the four-phase coexistence, which actually contradicts the phase rule of Gibbs, by taking into account gravity. Therefore, sedimentation of particles leads to a

(12) van der Beek, D.; Lekkerkerker, H. N. W. Langmuir 2004, 20, 8582-8586. (13) van der Beek, D.; Radstake, P. B.; Petukhov, A. V.; Lekkerkerker, H. N. W. Langmuir 2007, 23, 11343-11346.

(14) Mourad, M. C. D.; Byelov, D. V.; Petukhov, A. V.; de Winter, D. A. M.; Verkleij, A. J.; Lekkerkerker, H. N. W. J. Phys. Chem. B 2009, 113, 11604-11613.

(15) de Gennes, P. G.; Prost, J. The Physics of Liquid Crystals; Clarendon Press: Oxford, U.K., 1993; p 616.

(16) Asakura, S.; Oosawa, F. J. Polym. Sci. 1958, 33, 183-192.

(17) Zhang, S. D.; Reynolds, P. A.; van Duijneveldt, J. S. J. Chem. Phys. 2002, 117, 9947-9958.

(18) Wensink, H. H.; Lekkerkerker, H. N. W. Europhys. Lett. 2004, 66, 125.

(19) Tuinier, R.; Aarts, D. G. A. L.; Wensink, H. H.; Lekkerkerker, H. N. W. Phys. Chem. Chem. Phys. 2003, 5, 3707-3715.

(20) Luan, L.; Li, W.; Liu, S.; Sun, D. Langmuir 2009, 25, 6349-6356.

(21) Petukhov, A. V.; van der Beek, D.; Dullens, R. P. A.; Dolbnya, I. P.; Vroege, G. J.; Lekkerkerker, H. N. W. Phys. Rev. Lett. 2005, 95, 077801.

(22) van der Kooij, F. M.; Vogel, M.; Lekkerkerker, H. N. W. Phys. Rev. E 2000 $62,5397$. 


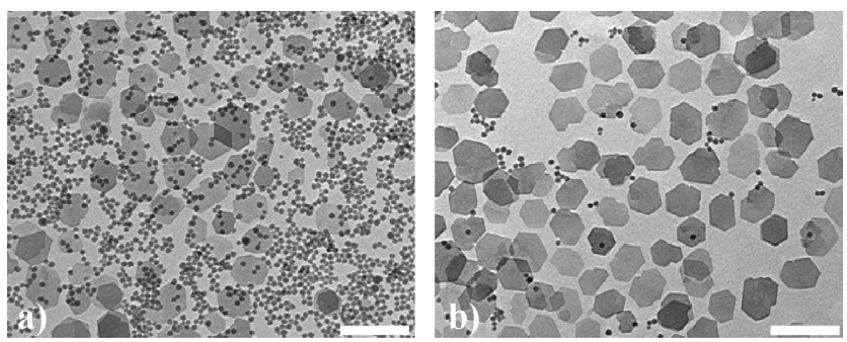

Figure 1. Transmission electron micrographs of samples taken from a phase separated silica-gibbsite mixture: a) upper phase, b) bottom phase. The scale bar denotes $200 \mathrm{~nm}$.

density gradient that facilitates the formation of multiple phases in a capillary of sufficient height.

So far, many studies of colloidal platelets were performed using model systems, which interacted through a hard-wall potential. The depletion interactions are usually studied using polymers as depletant agents. In this article, we explore the phase diagram of mixtures of charged gibbsite platelets and like-charged silica spheres in the low salinity regime. We chose colloidal spheres as depletants because they are more effective than polymers. ${ }^{23}$ Both of our colloidal species were small and charged enough, so sedimentation due to gravity was negligible. In contrast with the previously studied systems, ${ }^{20,22,24-26}$ which were in steady state because of external field, gravity, we will show here the phase behavior of mixed sphere/ platelet suspensions in equilibrium. The article is organized as follows. After a brief description of samples and instrumentation, an experimental phase diagram of mixtures of charged spheres and platelets will be presented. Furthermore, we will discuss in detail the structures formed in pure gibbsite and gibbsite/silica mixtures. The columnar lattice spacings and the nematic order parameters will be presented. At the end, we qualitatively compare our experimental phase diagram with the theoretical predictions available for mixtures of hard platelets and polymers.

\section{Materials and Methods}

Colloidal gibbsite platelets were synthesized according to the standard procedure described by Wierenga et al. ${ }^{8}$ After this, $\mathrm{Al}_{13}$ ions (as produced by hydrolysis of aluminum chlorohydrate, $\mathrm{Al}_{2}(\mathrm{OH})_{5} \mathrm{Cl}_{2} \cdot 3 \mathrm{H}_{2} \mathrm{O}$, Locron $\mathrm{P}$, Hoechst AG) were adsorbed onto the gibbsite surfaces to provide additional stabilization. ${ }^{8}$ Through a sequence of centrifugations $(24 \mathrm{~h}, 1800 G)$ and redispersions, excess of $\mathrm{Al}_{13}$ ions was removed, and the platelets were brought into the $10^{-4} \mathrm{M} \mathrm{NaCl}$ solution. Silica Ludox CL spheres was obtained from Sigma-Aldrich. The surface of Ludox CL was coated by the manufacturer with aluminum oxide, which converts the charge of the particles from the negative to the positive. Therefore, the surfaces of gibbsite platelets and silica spheres had the same charge. Ludox CL suspensions were stable at the used salt concentration and showed no traces of gelation.

Particle averaged diameter, $\langle D\rangle$, was determined from transmission electron microscopy (TEM) images (Figure 1). In the case of the gibbsite platelets, the particle diameter was defined as the diameter of an equal-area circle. Atomic force microscopy was used to obtain the thickness of the gibbsite platelets, $\langle L\rangle$. The particle characteristics are listed in Table 1.

(23) Kleshchanok, D.; Tuinier, R.; Lang, P. R. J. Phys.: Condens. Matter 2008 20,073101

(24) Hemmen, H.; Ringdal, N. I.; De Azevedo, E. N.; Engelsberg, M.; Hansen, E. L.; Meheust, Y.; Fossum, J. O.; Knudsen, K. D. Langmuir 2009, 25, 1250712515.

(25) Fonseca, D. M.; Meheust, Y.; Fossum, J. O.; Knudsen, K. D.; Parmar, K. P. S. Phys. Rev. E 2009, 79, 021402.

(26) van der Beek, D.; Schilling, T.; Lekkerkerker, H. N. W. J. Chem. Phys. 2004, 121, 5423-5426.
Table 1. Characteristic Sizes of Gibbsite Platelets and Silica Spheres Used in This Study

\begin{tabular}{llll}
\hline & $<D>/ \mathrm{nm}$ & $<L>/ \mathrm{nm}$ & \\
\hline gibbsite & $95.0 \pm 16.0$ & $10.6 \pm 3.7$ & $L / D=0.11$ \\
silica & $16.8 \pm 1.7$ & & $D_{\text {silica }} / D_{\text {gibbsite }}=0.18$
\end{tabular}

All suspensions were in $10^{-4} \mathrm{M} \mathrm{NaCl}$ solution. Concentrations of counterions in our studies could only be approximated because the number of surface charges, $z$, on the platelets and spheres is not known. In colloidal suspensions, both electrolyte and counterion concentrations have to be taken into account to calculate the ionic strength, IS. From the latter, one can calculate the Debye screening length as $\kappa^{-1}[\mathrm{~nm}]=0.304 /(I S)^{1 / 2}[\mathrm{~mol} / \mathrm{L}],{ }^{27}$ which will be reduced for the concentrated suspensions of strongly charged particles in comparison with the diluted suspensions containing the same amount of electrolyte.

The gravitational length of colloids can be calculated as $L_{\mathrm{g}}=$ $k_{\mathrm{B}} T / m g$, where $k_{\mathrm{B}}$ is the Boltzmann constant, $T$ is the absolute temperature, $m$ is buoyant mass, and $g$ is the gravitational acceleration. For the gibbsite platelets used in this study, $L_{\mathrm{g}}$ is $4.8 \mathrm{~mm}$. However, Rasa and Philipse showed that in concentrated colloidal suspensions with a low electrolyte concentration, particles should have an extended gravitational length due to charges at their surfaces. ${ }^{28,29}$ Indeed, no sedimentation could be observed in our suspensions of platelets even after a year of equilibration.

Samples were prepared by mixing the stock suspensions of gibbsite and silica with the solvent such that the desired colloid concentration was obtained. One series of mixed gibbsite/silica suspensions was made with the constant volume concentration of gibbsite $(12 \mathrm{vol} \%)$, and the amount of silica varied from 0.5 to 8.1 vol $\%$. Additionally, a sample with 9.3 vol \% gibbsite and $7.0 \mathrm{vol} \%$ silica was prepared to determine the tie lines in the experimental phase diagram. Mixtures for visual observations were put in the glass capillaries $(1.0 \times 10.0 \mathrm{~mm}$ cross section, Vitrotubes, VitroCom) that were flame-sealed. Suspensions for microradian X-ray diffraction $(\mu \mathrm{radXRD})$ experiments were placed in the round Mark tubes ( $2 \mathrm{~mm}$ diameter, $10 \mu \mathrm{m}$ wall thickness, W. Müller, Berlin). All samples were stored vertically at $20^{\circ} \mathrm{C}$ to reach phase equilibrium. The macroscopic phase separation took $24 \mathrm{~h}$. Once phase-separated, the samples were checked for liquid crystallinity with crossed polarisers and by microradian X-ray diffraction ( $\mu$ radXRD).

$\mathrm{X}$-ray scattering experiments were performed at the European Synchrotron Radiation Facility (ESRF) in Grenoble, France, at the Dutch-Belgian beamline BM-26 $6^{30}$ and the high energy materials science beamline ID-11. The so-called microradian X-ray diffraction ( $\mu$ radXRD) setup has been used. ${ }^{31}$ The X-ray beam was focused by a set of compound refractive lenses (CRLs) ${ }^{32}$ at a phosphor screen of the CCD (charge-coupled device) X-ray detectors (Photonic Science, $4008 \times 2671$ pixels of 22 and $9 \mu \mathrm{m}$ square). At the beamline BM-26, a set of beryllium CRLs was used. The capillaries were placed just after the CRLs at a distance of $\sim 8 \mathrm{~m}$ from the detector. This setup allowed us to achieve an angular resolution on the order of 5-7 microradians. The X-ray photon energies of $13 \mathrm{keV}$ (wavelength, $\lambda$, of $0.095 \mathrm{~nm}$ ) were used. At the beamline ID-11, an X-ray transfocator ${ }^{33}$ based on aluminum CRLs was used for beam focusing. The X-ray photon energies of $27 \mathrm{keV}(\lambda=0.046 \mathrm{~nm})$ were selected. The beam diameter in the sample was $\sim 0.5 \mathrm{~mm}$ in both setups.

27) Israelachvili, J. N. Intermolecular and Surface Forces, 2nd ed.; Academic Press: London, 1991; p 480

(28) Rasa, M.; Philipse, A. P. Nature 2004, 429, 857-860.

(29) Philipse, A. P. J. Phys.: Condens. Matter 2004, 16, S4051-S4062.

(30) Borsboom, M.; Bras, W.; Cerjak, I.; Detollenaere, D.; van Loon, G. D.; Goedtkindt, P.; Konijnenburg, M.; Lassing, P.; Levine, Y. K.; Munneke, B.; Oversluizen, M.; van Tol, R.; Vlieg, T. E. J. Synchrotron Radiat. 1998, 5, 518-520.

(31) Petukhov, A. V.; Thijssen, J. H. J.; ’t Hart, D. C.; Imhof, A.; van Blaaderen, A.; Dolbnya, I. P.; Snigirev, A.; Moussaid, A.; Snigireva, I. J. Appl. Crystallogr. 2006, 39, 137-144.

(32) Snigirev, A.; Kohn, V.; Snigireva, I.; Lengeler, B. Nature 1996, 384, 49-51.

(33) Snigirev, A.; Snigireva, I.; Vaughan, G.; Wright, J.; Rossat, M.; Bytchkov, A.; Curfs, C. J. Phys.: Conf. Ser. 2009, 186, 012073. 


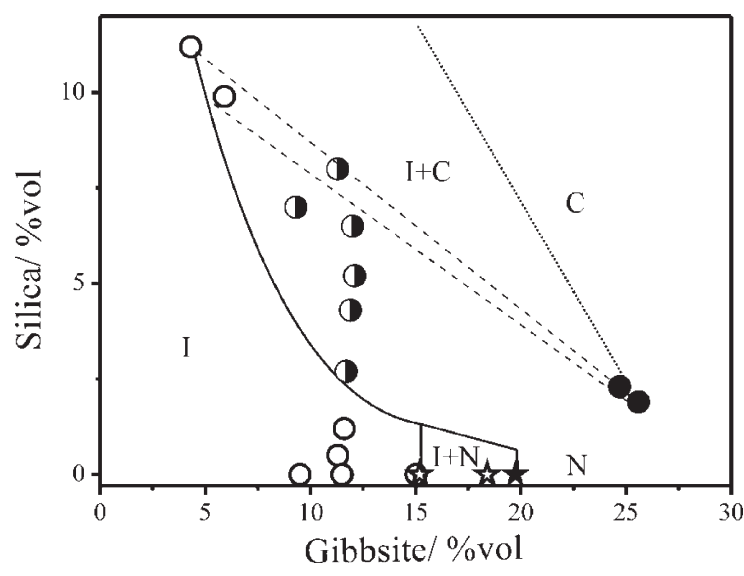

Figure 2. Experimental phase diagram of aqueous silica/gibbsite mixtures. Broken straight lines are the experimentally found tie lines, connecting coexisting phases. Phase boundaries depicted as solid curves were found from the experiment. The dotted line indicates the extrapolated columnar phase boundary.

\section{Experimental Phase Diagram of Aqueous Silica/ Gibbsite Mixtures}

III.A. Phase Behavior of Pure Gibbsite Suspensions. Using visual observations and $\mu \mathrm{radXRD}$, we determined structures in pure gibbsite and silica/gibbsite suspensions. A detailed analysis of the found structures will be given in Section IV of this article. Here we present an experimental phase diagram of aqueous silica/gibbsite mixtures (Figure 2). Pure gibbsite suspensions were found to be isotropic $(O)$ and showed no birefringence up to a platelet volume concentration of $15.2 \mathrm{vol} \%$. Upon increasing the platelet volume concentration further, we observe an isotropic/nematic phase coexistence ( $)$. Samples with pure nematic phase were not prepared because the suspensions with the concentrations of gibbsite larger than $18.4 \mathrm{vol} \%$ become very viscous. To estimate the nematic phase boundary, we extrapolated the relative volume of the nematic phase from two samples showing an isotropic/nematic coexistence to $100 \%$, which gave a value for the concentration of platelets of $20 \mathrm{vol} \%$, denoted as $\star$ in Figure 2. A columnar phase was not detected in pure gibbsite samples.

III.B. Phase Behavior of Silica/Gibbsite Mixtures. Small amounts of silica spheres (concentrations $<1.7 \mathrm{vol} \%$ ) added to $12 \mathrm{vol} \%$ isotropic suspension of gibbsite did not change its phase behavior. However, when the amount of spheres was larger than $2.7 \mathrm{vol} \%$, an isotropic/columnar phase transition was induced (denoted as in Figure 2). Photographs of the phase-separated samples observed between crossed polarisers are presented in Figure 3. The relative amount of the columnar phase in the samples increased linearly with the increasing amount of silica, depicted in Figure 4. From this linear dependence, we can estimate at which silica concentration a pure columnar phase occurs at given gibbsite concentration (dotted line in Figure 2).

The tie lines in the phase diagram (broken straight lines in Figure 2) connecting coexisting phases were found from the quantitative analysis of two samples with an isotropic/columnar phase coexistence $(9.3 \mathrm{vol} \%$ gibbsite $/ 7.0 \mathrm{vol} \%$ silica and $11.3 \mathrm{vol} \%$ gibbsite $/ 9.0$ vol $\%$ silica). After the macroscopic phase separation proceeded, the columnar and the isotropic phases of these samples were analyzed quantitatively with respect to the amount of gibbsite, silica, and water in them. TEM micrographs (such as Figure $1 \mathrm{a}, \mathrm{b})$ were used to find the number ratio of gibbsite platelets to silica spheres in the isotropic and columnar phases. Gravimetric analysis was used to determine the amount of water and, consequently, of the total solid compounds (gibbsite and silica) in two phases. The volume fractions of gibbsite and silica in the isotropic and columnar phases were calculated using the particle number ratio, the amount of the total solid compounds,

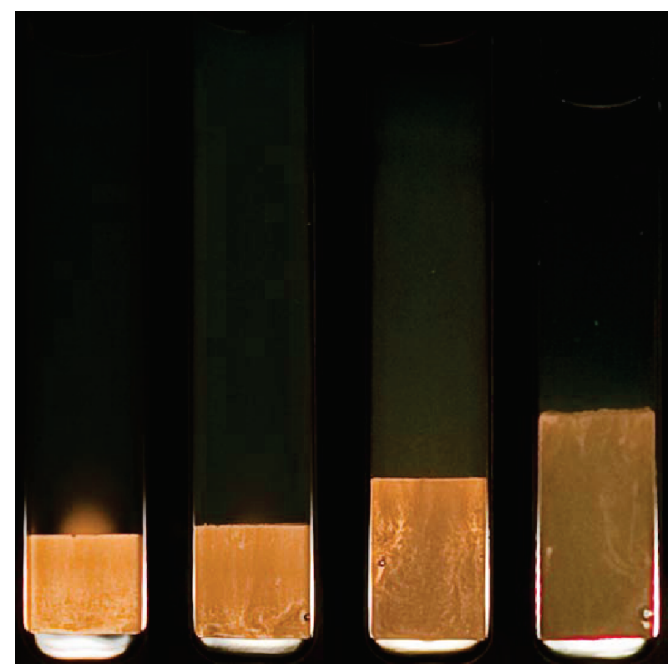

Figure 3. Mixed silica/gibbsite suspensions observed between crossed polarisers. Concentration of gibbsite in all samples is 12 vol \%. Concentration of silica spheres increases from the left to the right as $4.3,5.2,6.5$, and $8.1 \mathrm{vol} \%$.

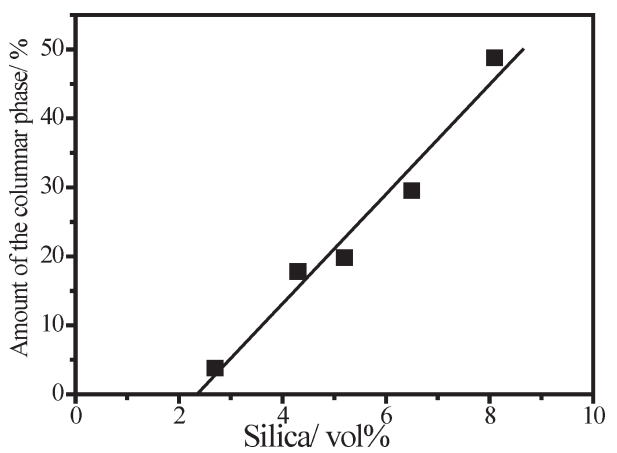

Figure 4. Amount of the columnar phase in mixtures of gibbsite platelets and silica spheres as a function of the spheres concentration. The straight line is a fit indicating the linear increase of the amount of the columnar phase with the increasing concentration of spheres.

and the particle averaged diameters and thickness. We obtained the tie lines by joining the composition points corresponding to the isotropic and columnar phases being in coexistence.

Figure 1 presents the TEM micrographs of (a) an isotropic top and (b) a columnar bottom phase of a sample with the isotropic/ columnar phase coexistence. The micrographs already point out that most of the silica spheres are located in the isotropic phase, whereas the gibbsite platelets preferably accumulate in the columnar phase. This observation is corroborated by the quantitative analysis. However, the columnar phase is not fully free of silica, and thus up to $2.5 \mathrm{vol} \%$ of spheres is present in the bottom columnar phase (see Figure 2). In Section 4, we will speculate about the exact location of spheres in the columnar phase on the basis of a detailed analysis of the $\mu \mathrm{radXRD}$ data, which provide lattice spacings in the columnar phase.

\section{Structures in Gibbsite and Silica/Gibbsite Suspensions}

IV.A. Pure Gibbsite Suspensions: Isotropic and Nematic Phases. To verify the nature of the phases present in gibbsite and silica/gibbsite suspensions and to get a deeper insight into their structure, we performed high-resolution $\mu \mathrm{radXRD}$ measurements. First, we studied a reference sample containing only gibbsite particles at $12 \mathrm{vol} \%$ because this concentration was selected for all mixed silica/gibbsite suspensions. Visual observations proved this 


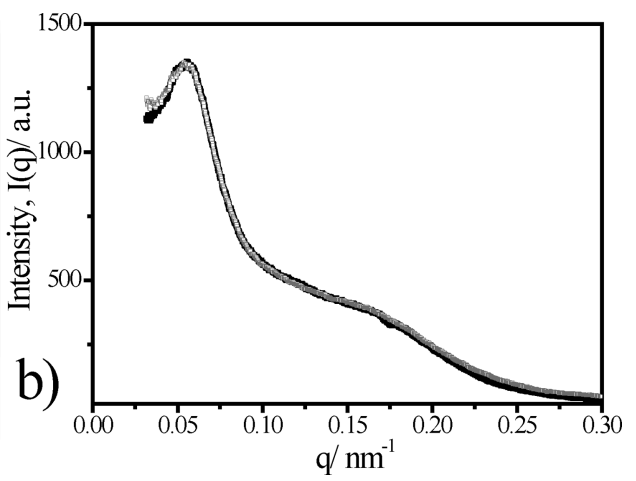

Figure 5. (a) 2D scattering pattern and (b) the intensity profiles along the $x$ (black solid curve) and $y$ (gray dotted curve) axes obtained from the isotropic gibbsite suspension of $12 \mathrm{vol} \%$.

sample to be isotropic because no birefringence was seen (not presented here). Figure 5a shows a $\mu$ radXRD $2 \mathrm{D}$ scattering pattern obtained from this sample. The scattering vector $(q)$-dependent intensity profiles, $I(q)$, along the $x$ and $y$ axes were extracted from the $2 \mathrm{D}$ scattering pattern by integrating a small wedge along the respective axis and are presented in Figure 5b. Here $q$ is determined by the scattering angle $2 \theta$ as $q=4 \pi(\sin \theta) / \lambda$.

As can be seen in Figure 5a, the scattering pattern is isotropic. The profiles of $I(q)$ verify the isotropic nature of the sample by being almost identical in the $x$ (solid curve) and $y$ (dotted curve) directions (Figure $5 b$ ). The intensity profiles $I(q)$ reveal a relatively broad peak at low $q$ and a broad shoulder at higher $q$. The first peak can be attributed to the maximum in the structure factor, $S(q)$, due to the side-to-side interparticle correlations. The shoulder is most likely due to a superposition of the decay of the form factor of the platelet, $F(q)$, and a maximum of $S(q)$ due to face-to-face interparticle correlations. ${ }^{34,35}$ The position, width, and height of the peak and the shoulder in $I(q)$ are nearly the same for all measured vertical positions along the capillary, indicating that the suspension consists of only one homogeneous isotropic phase. From the position of the maximum of $S(q)$, it is possible to find the mean nearest-neighbor separation between the centers of particles ${ }^{36}$

$$
d=\frac{2 \pi}{q_{\max }}
$$

where $q_{\max }$ is the $q$ at the peak position. Therefore, the separation distance between the platelets in the isotropic suspension of gibbsite is $116 \mathrm{~nm}\left(q_{\max }=0.054 \mathrm{~nm}^{-1}\right)$, which is larger than the diameter of the gibbsite platelet, $D_{\text {gibbsite }}=95.0 \mathrm{~nm}$.

Increasing the concentration of gibbsite platelets in the suspensions leads to the formation of a nematic phase. To characterize the structure of the nematic phase in detail, we performed $\mu \mathrm{radXRD}$ measurements on a sample containing $18.4 \mathrm{vol} \%$ of platelets. This sample was the most concentrated pure gibbsite sample used in our studies, and it showed an isotropic/nematic phase coexistence. Figure 6 a shows the sample between crossed polarisers. The upper phase is isotropic and showed no birefringence (not presented here). This is verified by the $\mu \mathrm{radXRD} 2 \mathrm{D}$ scattering patterns, which are isotropic and very similar to the reference sample in Figure 5. The bottom nematic phase reveals

(34) Lemaire, B. J.; Panine, P.; Gabriel, J. C. P.; Davidson, P. Europhys. Lett. 2002, 59, 55-61.

(35) van der Beek, D.; Petukhov, A. V.; Davidson, P.; Ferre, J.; Jamet, J. P.; Wensink, H. H.; Vroege, G. J.; Bras, W.; Lekkerkerker, H. N. W. Phys. Rev. E 2006, 73, 041402 .

(36) Pusey, P. N. Introduction to Scattering Experiments. In Neutrons, X-Ray and Light: Scattering Methods Applied to Soft Condensed Matter, Lindner, P., Zemb, T., Eds.; Elsevier: Amsterdam, 2002; p 541. three regions with or without birefringence between crossed polarisers. A thin region close to the tube bottom is birefringent. It is followed by a large region, which appears black. The top part of the nematic phase is birefringent again. The difference in the polarization behavior can be explained by different orientation of the platelets. To obtain the information about the orientation of platelets in the nematic phase, we performed $\mu \mathrm{radXRD}$ measurements at different vertical positions in the sample. The capillaries used for XRD measurements were round Mark tubes. Unfortunately, it was not possible to take a good quality picture of them because of high curvature of the walls. Figure 6a shows a sample in a flat capillary used for visual observations. The 2D scattering patterns from three different positions in the nematic phase are presented in Figure $6 b-d$.

The patterns in Figure 6b,d were measured at the top and at the bottom of the nematic phase, respectively. In these regions, the birefringence can be easily seen in Figure 6a. The pattern in Figure $6 \mathrm{c}$, was obtained from the middle part of the nematic phase, which appeared black between crossed polarisers. The scattering patterns in Figure 6b,d are very similar to each other and significantly differ from the pattern in Figure 6c. The difference between these patterns lies in the pronounced anisotropy of the patterns in Figure 6b,d, whereas Figure 6c looks entirely isotropic. To quantify these observations, intensity profiles, $I(q)$, along the $x$ and $y$ axes were extracted from all scattering patterns. The plots of $I(q)$ are presented in Figure $6 \mathrm{e}-\mathrm{g}$, where the intensity profiles in Figure 6e,g are, as expected, very similar to each other. A significant difference between the intensity profiles in $x$ and $y$ direction can be observed there. The profiles in Figure $6 \mathrm{f}$ of both $x$ and $y$ axes are identical, reflecting the isotropy of the scattering pattern in Figure 6c. Furthermore, the intensity profiles in Figure 6f are identical to the profiles in Figure 6e,g taken in the $x$ direction. They all reveal a strong peak at low $q$, whereas the intensity profiles in Figure 6e,g for the $y$ axis show an additional pronounced peak at high $q$. This shows that in the middle of the nematic phase the plates are orientated with their faces toward the cell wall. In other words, the X-ray beam detects the full faces of the platelets and can measure only the positional side-to-side correlation distance. The shorter face-to-face correlations are not seen because they are simply along the beam. Moreover, the region appears black in Figure 6a not because it is isotropic but because the light propagates along the optical axis there. On the other hand, in the top and bottom parts of the nematic phase, the platelets align with their horizontal director perpendicular to the surface; this orientation additionally makes their sides detectable in the direction of observation. Such orientation gives two length scales in the $\mu \mathrm{radXRD} 2 \mathrm{D}$ scattering patterns; that is, in addition to the sharp reflection at a small 

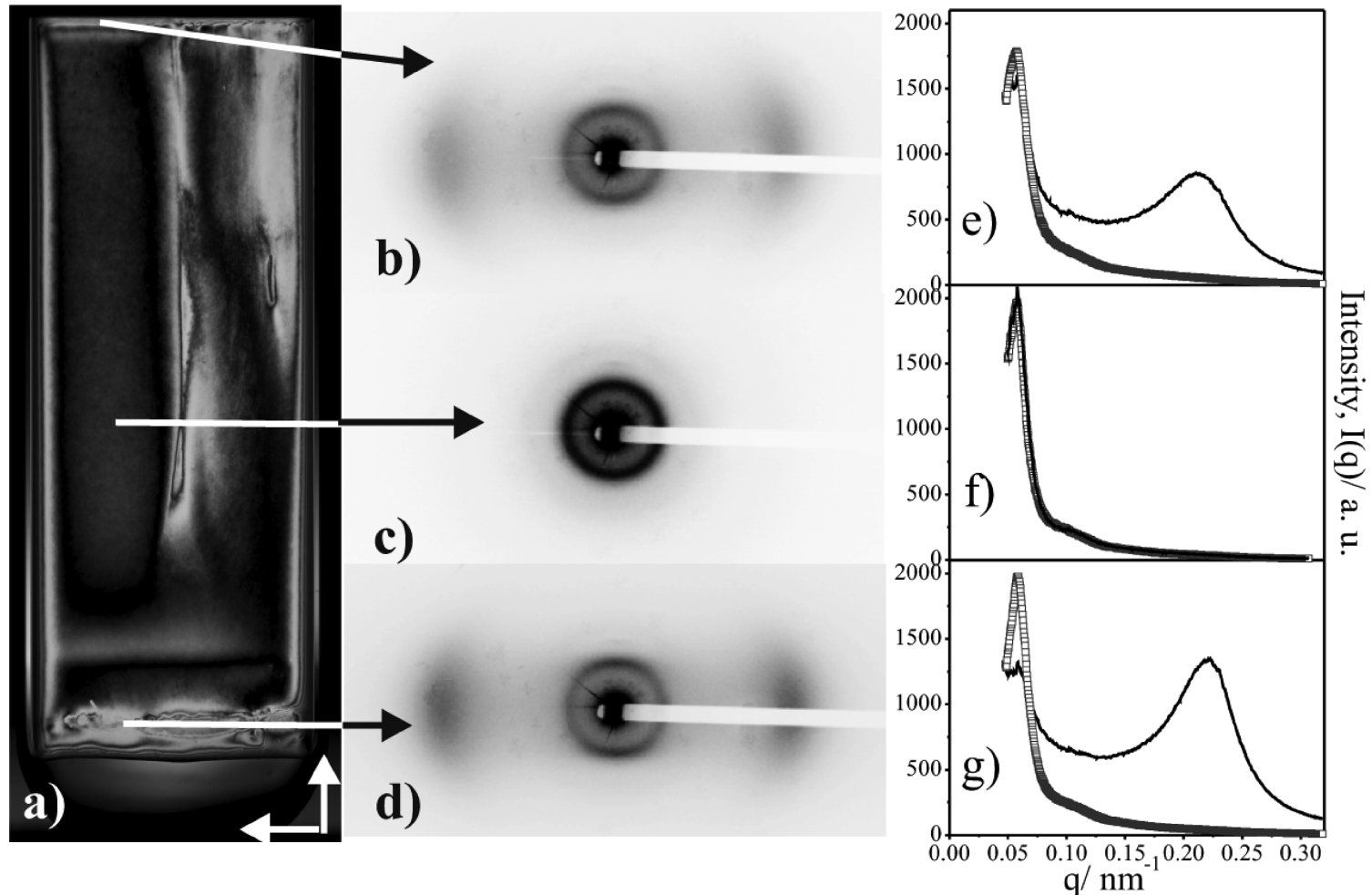

Figure 6. (a) Nematic phase of the suspension with $18.4 \mathrm{vol} \%$ of gibbsite observed between crossed polarisers (their orientation is indicated at the bottom). (b-d) The $\mu \mathrm{radXRD} 2 \mathrm{D}$ scattering patterns taken at different vertical positions in the nematic phase and (e-g) the corresponding intensity profiles along the $x$ (solid curves) and $y$ (dotted curves) axes.

Table 2. Peak Positions and Corresponding Side-to-Side and Face-toFace Correlation Distances between the Platelets Measured at the Different Positions of the Nematic Phase

\begin{tabular}{lcccc}
\hline $\begin{array}{c}\text { nematic } \\
\text { phase }\end{array}$ & $\begin{array}{c}\text { peak at } \\
\text { low } q\left(\mathrm{~nm}^{-1}\right)\end{array}$ & $\begin{array}{c}\text { side-to-side } \\
\text { correlation } \\
\text { distance }(\mathrm{nm})\end{array}$ & $\begin{array}{c}\text { peak at } \\
\text { high } q\left(\mathrm{~nm}^{-1}\right)\end{array}$ & $\begin{array}{c}\text { face-to-face } \\
\text { correlation } \\
\text { distance }(\mathrm{nm})\end{array}$ \\
\hline $\begin{array}{l}\text { bottom } \\
\text { middle }\end{array}$ & 0.058 & 108 & 0.22 & 29 \\
top & 0.057 & 110 & \multicolumn{2}{c}{ not detected } \\
\end{tabular}

angle, a perpendicular broader reflection at a wider angle regime appears. Indeed, it can be nicely observed in Figure 6b,d. Furthermore, at this orientation, the light partially passes through the cross polarisers, as is indeed evident from Figure $6 \mathrm{a}$.

The visual observations of the three regions in the nematic phase are reproducible, and the X-ray patterns comply with the optical observations indicating that the formed regions will always appear. It appears that in the nematic phase the platelets prefer to position themselves with their faces toward the walls. Because the platelet is an anisotropic particle, it is not known how such a particle will orientate in junctions (in our case, at the top and the bottom of the nematic phase). This is why at these regions a different orientation of the platelets is observed.

The side-to-side and face-to-face correlation distances between the platelets can be calculated from the positions of the peak in $I(q)$ at low and high $q$, respectively, using eq 1 . Table 2 presents the side-to-side and face-to-face correlation distances at the different positions of the nematic phase.

By looking at the azimuthal intensity distribution at the $q$ peak values corresponding to the face-to-face correlations, we can find the tilt angle of the platelet side in respect to the vertical axis, $\omega_{0}$. Additionally, the order parameter can be investigated in the bottom and top regions of the nematic phase. As shown in Figure $6 \mathrm{~b}, \mathrm{~d}$, the scattering patterns from these regions reveal

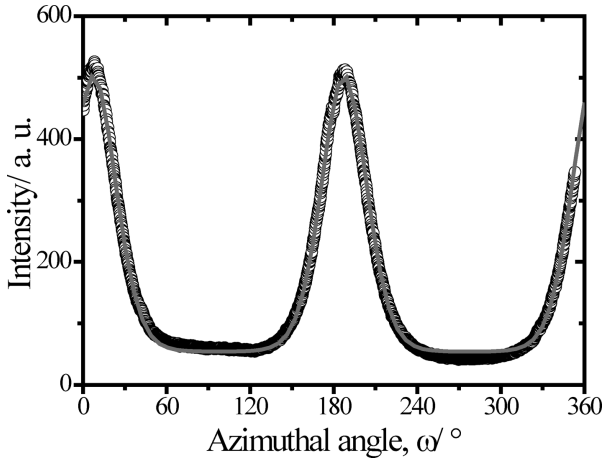

Figure 7. Intensity as a function of the azimuthal angle at $q=$ $0.056 \mathrm{~nm}^{-1} 3 \mathrm{~mm}$ from the nematic-isotropic interphase. The gray line is the fit to eq 2 .

structure peak at high $q$, which can be used to determine the order parameter for each position in the system. Figure 7 presents the azimuthal intensity distribution at the $q=0.21 \mathrm{~nm}^{-1}$, where this peak occurs, extracted from the 2D scattering pattern measured 3 $\mathrm{mm}$ from the nematic-isotropic interphase. Similar distributions were extracted for all scans in the nematic phase. In its bottom and top parts, all distributions show features similar to those in Figure 7, whereas the middle part is isotropic.

Two things are clear from this plot. First, the scattering intensity is very anisotropic. Second, the intensity peaks are not aligned along the $x$ axis but slightly turned, indicating a small tilt angle of the platelets. We now apply the formalism used by Purdy et al. ${ }^{37}$ to determine the nematic order parameter, $S_{2}$. First, we fit the azimuthal intensity distribution

(37) Purdy, K. R.; Dogic, Z.; Fraden, S.; Ruhm, A.; Lurio, L.; Mochrie, S. G. J. Phys. Rev. E 2003, 67, 031708. 


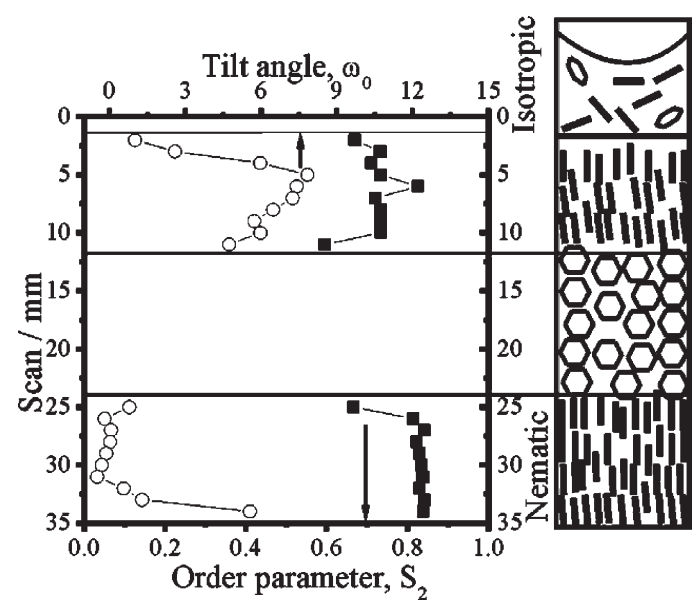

Figure 8. Tilt angle $(\bigcirc)$ and the order parameter $S_{2}(\boldsymbol{\square})$ for different sample height scans. Error bars correspond to the symbol size.

with the following function

$$
I=\text { baseline }+I_{0} f(\omega)
$$

Here $I_{0}$ is the normalizing factor, $\omega$ is the azimuthal angle, and $f(\omega)$ is the orientational distribution function described as

$$
f(\omega)=\exp (-\alpha P(\omega))
$$

where $\alpha$ is the width of $P(\omega)$, which in our case is a Legendre polynomial

$$
P(\omega)=0.5\left(3 \cos ^{2}\left(\omega-\omega_{0}\right)-1\right)
$$

here $\omega_{0}$ is the tilt angle. The function $P(\omega)$ can be arbitrarily chosen, as has been shown by Prudy et al.; ${ }^{37}$ however, it has to be able to fit the azimuthal intensity distribution. Furthermore, the order parameter $S_{2}$ can be determined as follows

$$
S_{2}=\frac{\int f(\omega) P(\omega) \sin (\omega) \mathrm{d} \omega}{\int f(\omega) \sin (\omega) \mathrm{d} \omega}
$$

In Figure 8, the order parameter $(\mathbf{\square})$ and the tilt angle, $\omega_{0}(\bigcirc)$, are shown for different sample height positions.

The large $q$ peak is not visible in the middle region of the nematic phase (Figure $6 \mathrm{c}$ ), and it is therefore not possible to extract any tilt angle or order parameter in this region. The analysis reveals that the platelets have their horizontal director perpendicular to the beam at least a centimeter from both the top and bottom surfaces (schematically shown in Figure 8). Moreover, it was possible to detect that the platelets are also slightly tilted. The platelets are very well aligned through the nematic phase, giving an order parameter $>0.7$. Its value is only slightly lower than 0.85 observed in a nematic phase of magnetically oriented gibbsite platelets. ${ }^{38}$ An interesting observation is that the order parameter is larger at the bottom part, indicating that the sample is slightly denser there. Additionally, as shown in Table 2, both the side-to-side and the face-to-face correlation distances slowly decrease from the top to the bottom of the nematic phase. The gibbsite platelets at the bottom are more compressed because of a gradient in the osmotic pressure.

IV.B. Mixed Silica/Gibbsite Suspensions: Columnar Phase. The addition of silica spheres to the isotropic gibbsite suspension of $12 \mathrm{vol} \%$ induced a phase separation when the amount of silica reached $2.7 \mathrm{vol} \%$. The upper phase showed no

(38) van der Beek, D.; Davidson, P.; Wensink, H. H.; Vroege, G. J.; Lekkerkerker, H. N. W. Phys. Rev. E 2008, 77, 031708. birefringence and was determined to be isotropic. The bottom phase appeared birefringent (Figure 3); however, it showed no optical Bragg reflections. The absence of optical Bragg reflections did not necessarily mean that the observed liquid crystalline phase is nematic. It could also be explained by the fact that the separation distances between the gibbsite platelets are so small so that the wavelength of the reflected light lies outside of the visible light range. This means that the visual observations could not provide the definitive information about the structure of the liquid crystal phase in our mixed silica/gibbsite suspensions. Therefore, $\mu \mathrm{radXRD}$ measurements had to be performed to clarify the structure of the bottom phase in a mixed suspension of silica and gibbsite. We studied a sample with the highest amount of silica (8.1 vol \% silica, 12.2 vol $\%$ gibbsite). The $2 \mathrm{D}$ scattering pattern and the $I(q)$ along the $x$ (black solid curve) and $y$ (gray dotted curve) axes of the bottom phase are shown in Figure 9a,b, respectively. The scattering pattern and the $I(q)$ of the isotropic phase are not presented here because they were identical to those shown in Figure 5.

Four sharp rings at small angles appear in the scattering pattern of the bottom phase. They correspond to four sharp peaks in the intensity profile and are best seen in a profile taken along the $x$ direction (black curve). The relative positions of the peaks obey the relationship $1: \sqrt{ } 3: \sqrt{ } 4: \sqrt{ } 7$. Therefore, the peaks can be attributed to (100), (110), (200), and (210) Bragg reflections. This indicates that the sample has hexagonal structure of columnar stacks. Differences in $I(q)$ in $x$ and $y$ directions are due to the orientation of the platelets in relation to the incident X-ray beam. The indexing of the scattering data was assessed by plotting $q_{h k l}$ vectors $(h k l$ the Miller indices) of the Bragg peaks versus $\left(h^{2}+h k+k^{2}\right)^{1 / 2}$ (see Figure 10). For a hexagonal structure, such a plot should pass through the origin and be linear with the slope, $b$, of

$$
b=\frac{4 \sqrt{3} \pi}{3} \frac{1}{a_{\mathrm{d}}}
$$

where $a_{\mathrm{d}}$ is the hexagonal lattice spacing. ${ }^{39,40}$ The line in Figure 10 indeed indicates a very good fit of the scattering data to the hexagonal structure. The value of $a_{\mathrm{d}}$, obtained from the slope of the fit line in Figure 10 is $133 \mathrm{~nm}$. Lattice spacings, $a_{\mathrm{L}}$, revealing face-to-face positional correlations between platelets in columns can be calculated from the $q_{001}$ value $\left(q_{001}=0.263 \mathrm{~nm}\right)$ as $a_{\mathrm{L}} \equiv 2 \pi /$ $q_{(001)}$. The value of $a_{\mathrm{L}}$ is $24 \mathrm{~nm}$. Furthermore, we measured the width of the $q_{(001)}$ peak, which is $0.02 \mathrm{~nm}^{-1}$. On the basis of the Scherrer equation, ${ }^{41}$ we can estimate that the positional correlations in columns extend over at least 13 lattice periods. The position of all peaks slightly shifts to the larger $q$ as the sample is scanned from the top to the bottom of the columnar phase. This indicates that both the side-to-side and the face-to-face correlation distances between platelets slowly decrease. An interesting observation from the $\mu \mathrm{radXRD} 2 \mathrm{D}$ scattering patterns of the high $q$ peaks in the nematic (Figure 6b,d) and columnar phases (Figure 9a) is that the orientation of the platelets in those phases differs by $90^{\circ}$. The columnar stacks are perpendicular to the bottom of the tube, whereas in the nematic phase, platelets are oriented with their horizontal director parallel to the bottom of the tube.

As we pointed out in Section III, up to $2.5 \mathrm{vol} \%$ of silica spheres is present in the bottom columnar phase (see the phase diagram Figure 2). On the basis of geometrical reasons, we can speculate about the exact location of spheres in the columnar phase. The face-to-face correlation distance between the platelets

(39) Hahn, T. International Tables for Crystallography; Reidel: Dortrecht, The Netherlands, 1983

(40) Holmqvist, P.; Alexandridis, P.; Lindman, B. Langmuir 1997, 13, 2471-2479.

(41) Warren, B. E. The X-ray Diffraction; Dover Publications: New York, 1990; p 381. 

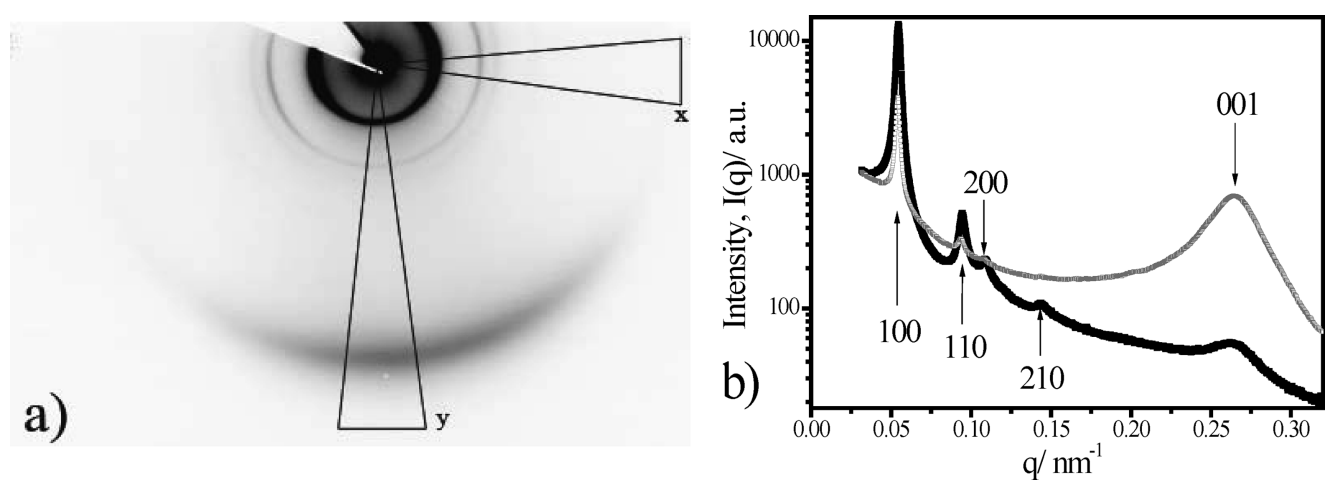

Figure 9. (a) $2 \mathrm{D}$ scattering pattern and (b) the intensity profiles along the $x$ (black solid curve) and $y$ (gray dotted curve) axes obtained from the silica/gibbsite mixture (12.2 vol \% gibbsite, $8.1 \mathrm{vol} \%$ silica).

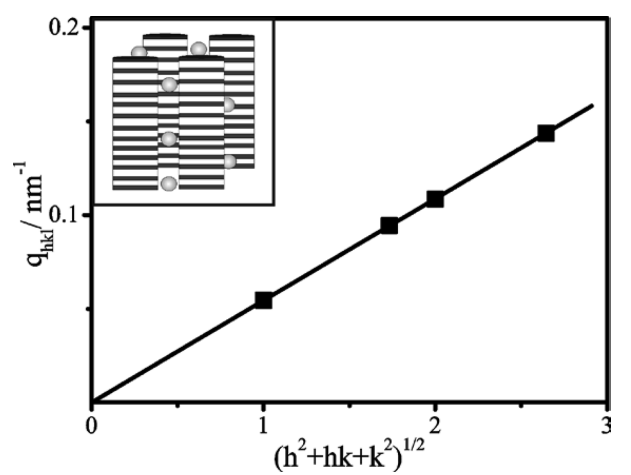

Figure 10. $q$ values of Bragg reflections of Figure 9 versus $\left(h^{2}+h k\right.$ $\left.+k^{2}\right)^{1 / 2}$. The straight line passing through the origin indicates a very good fit of the scattering reflections to the hexagonal structure. The inset depicts the possible location of the spheres in the platelets columnar phase.

in columns, $a_{\mathrm{L}}$, was found to be $24 \mathrm{~nm}$. This value is smaller than the sum of the platelet thickness and the sphere diameter. Therefore, we can conclude that the spheres are not situated between faces of the platelets in the stacks of columns. The hexagonal lattice spacing of the columnar phase, $a_{\mathrm{d}}$, found from $\mu \mathrm{radXRD}$ is $133 \mathrm{~nm}$. This value is larger than the sum of diameters of the sphere and the platelets, which is equal to $111.8 \mathrm{~nm}$. Therefore, it follows that the spheres can be located only in the regions between the columns of platelets (depicted as an inset in Figure 10).

\section{Discussion and Conclusions}

On the basis of visual observations and $\mu \mathrm{radXRD}$ measurements, we presented an experimental phase diagram of aqueous silica/gibbsite mixtures in the low salinity regime. Here we will compare this experimental phase diagram with the previous experimental and theoretical studies on platelet suspensions. Silica spheres added to gibbsite suspensions acted as depletants and changed the phase behavior of gibbsite platelets. Whereas pure gibbsite suspensions showed the isotropic phase and the isotropic/nematic phase coexistence, the mixed suspensions of spheres and platelets showed isotropic/columnar phase coexistence in a very broad colloidal concentration region. This tendency of platelets to form columnar stacks when a depletant agent is added was shown by Mason on an example of wax disks and surfactant micelles. ${ }^{42}$ Moreover, he theoretically showed that the contact value of the depletion attraction is the largest when the disks approach each other face-to-face, so columns are formed.

(42) Mason, T. G. Phys. Rev. E 2002, 66, 060402.
In agreement with this finding, our $\mu \mathrm{radXRD}$ measurements show that in the columnar phase silica spheres are not located between the faces of gibbsite platelets (which would be energetically less favorable) but occupy the regions between the stacks (depicted as an inset in Figure 10). Therefore, only the isotropic phase and the isotropic/columnar phase coexistence were found in mixed silica/gibbsite suspensions. This finding is different from the observations of van der Kooij et al., ${ }^{22}$ where four phases were found in mixtures of hard platelets and ideal polymers depletants. However, we can explain the difference by the fact that sedimentation played an important role in the study of van der Kooij et al. because an estimated gravitational length of hard gibbsite platelets used in their study is $0.9 \mathrm{~mm}$, which is smaller than the container length. Wensink et al. ${ }^{18}$ showed theoretically that sedimentation indeed leads to rich phase behavior of platelets, such as the four phase equilibrium, which is not present in zerogravity case and contradicts the phase rule of Gibbs. Gibbsite platelets used in our study were small and charged, which means that their gravitational length significantly increased. ${ }^{28,29}$ This led to the negligible sedimentation in our colloidal system. Therefore, the phase behavior presented here for mixtures of charged silica and gibbsite is in a "true" equilibrium, which explains the absence of four phase coexistence.

We would also like to compare our experimental phase diagram with the theoretical predictions. However, to our knowledge, there is no theoretical work published for mixtures of charged spheres and platelets. Therefore, we can only qualitatively compare our experimental phase diagram with the existing simulations of Zhang et al. ${ }^{17}$ on the mixtures of hard platelets and ideal polymers. In the simulations, the $L / D$ ratio was 0.1 , and the ratio $D_{\text {polymer }} / D_{\text {gibbsite }}$ equaled 0.5 ; gravity was negligible. The size ratios used in the simulations are slightly different from our values (Table 1), also due to the Debye screening length changing the effective size of our colloids. However, because we do not have simulation data for the colloid size ratios used in our studies, we use the latter ratios for comparison. Simulations show that low concentrations of depletant do not change the phase behavior of the mixture. Therefore, isotropic, nematic, and columnar phases should be present. At high depletant concentration, the depletion attraction is strong enough to induce an isotropic/columnar phase transition without the occurrence of a nematic phase. These theoretical predictions were partially observed by us experimentally. Therefore, the addition of silica spheres, when the concentration of spheres was $>2.7 \mathrm{vol} \%$, affected the phase diagram of the gibbsite suspensions significantly. The most striking effect was, indeed, the appearance of a broad isotropic/columnar phase coexistence region (Figure 2). However, we were not able to find a nematic phase in mixtures with low sphere concentrations. 
The reason could be due to the small area of the nematic phase on the phase diagram of platelet/sphere mixtures. Additionally, the work in the high concentration region of platelets was difficult because of the large viscosity of the gibbsite suspensions. Our future study will be to obtain a theoretical phase diagram for mixtures of charged platelets and spheres to compare the exact location of the phase boundaries.

To conclude, rich information was obtained from $\mu \mathrm{radXRD}$ measurements not only about the structure of the phases in mixed sphere/platelets suspensions but also about the orientation of the platelets in liquid crystalline phases. This in combination with the thorough phase diagram analysis gives a deeper insight into the physics of anisotropic mixed systems. So far, we studied silica and gibbsite mixtures in the low salinity regime. The electrostatic repulsion between colloids is important in this system, affecting the volume of particles and the size ratios $D_{\text {silica }} / D_{\text {gibbsite }}$ and $L / D$ due to the Debye screening length. Additionally, the electrostatic repulsion increases the gravitational length of colloids, keeping the system in equilibrium in difference with many previous studies. Certainly, an addition of salt will change the interactions affecting both the structures and the phase behavior of the system. Studying the role of salt concentration will be an interesting subject for further investigations.

Acknowledgment. We thank Mark Vis for the AFM measurements and photographs of the samples and Anke Leferink op Reinink and Jan Hilhorst for the $\mu \mathrm{radXRD}$ measurements at the beamline ID-11. Maurice Mourad is acknowledged for the synthesis of gibbsite. Remco Tuinier and Albert Philipse are thanked for the fruitful discussions. The team of BM-26 and Gavin Vaughan from the ID-11 are thanked for their assistance during the $\mu \mathrm{radXRD}$ measurements. The work of D.K. was financed by the "Stichting voor Fundamenteel Onderzoek der Materie (FOM)". 